\title{
ON THE SYNTHESIS OF DOL GROWTH
}

\author{
KEIJO RUOHONEN
}

\section{Introduction}

In 1968 A. Lindenmayer [3] introduced mathematical models for the development of certain filamentous organisms. These models (now customarily called $L$ systems or developmental systems) are essentially generative grammars with parallel rewriting. Each cell type of the organism is denoted by a symbol. The stages of development of the organism then correspond to certain strings of these symbols.

In subsequent years L systems have been one of the most vigorously studied subjects in formal language theory. Among the most widely studied L systems are the DOL systems. The "D" means that the development is deterministic, i.e. each stage has a unique successor; the " $\mathrm{O}$ " means that there is no interaction between the cells of the described organism. The system being deterministic we can attach to each of the successive stages (starting from some initial stage) the unique number of the symbols of the corresponding string. Thus we get a function from $N$ ( $N$ is the set of nonnegative integers) into $N$. These functions are called growth functions. The basic papers in the field of growth functions are Szilard [7], Doucet [2], Salomaa [6], Paz and Salomaa [5] and Vitányi [9].

Our purpose is to state some sufficient conditions for a function (usually given by its generating function) to be a DOL growth function. Our results are extensions of those of Doucet [2] and Szilard [7].

We expect the reader to be familiar with the basic concepts of formal language theory, developmental systems theory (mainly for motivation) and the theory of matrices, linear difference equations and generating functions.

\section{Definitions and basic properties}

By an alphabet we mean a nonempty finite set. For an alphabet $A$ we denote by $A$ * the free monoid generated by $A$. The identity element of 
$A^{*}$ is denoted by $\lambda$. The length function $\lg$ is a homomorphism from $A^{*}$ onto the additive monoid $N$ such that $\lg (a)=1$ for each $a \in A$.

Definition 1. A deterministic Lindenmayer system without interaction (a DOL system in short) is an ordered triple $G=(A, \delta, \omega)$ where $A$ is an alphabet, $\delta: A^{*} \rightarrow A^{*}$ is a homomorphism (the production function) and $\omega \in A^{*}$ (the axiom). $G$ is called propagating (a PDOL system in short) if $\omega \neq \lambda$ and $\delta(P) \neq \lambda$ for all $P \in A^{*}$ such that $P \neq \lambda$. The growth function $f_{G}: N \rightarrow N$ of $G$ is defined by

$$
f_{G}(n)=\lg \left(\delta^{n}(\omega)\right) \quad(\text { for } n \in N) .
$$

Example 1. Consider the PDOL system $G=(\{a\}, \delta, a)$ where $\delta$ is given by $\delta(a)=a^{2}$. Then obviously $f_{G}(n+1)=2 f_{G}(n) \quad($ for $n \in N)$ and so $f_{G}(n)=2^{n}$ (for $\left.n \in N\right)$.

Let $G=(A, \delta, \omega)$ where $A=\left\{a_{1}, \ldots, a_{k}\right\}$ be a DOL system and $\mathrm{\rho}_{i}: A^{*} \rightarrow A^{*}$ be the homomorphism given by

$$
\left.\rho_{i}\left(a_{i}\right)=a_{i}, \rho_{i}\left(a_{j}\right)=\lambda \quad \text { (for } i, j=1, \ldots, k ; i \neq j\right) .
$$

Then the row vector $\pi_{G}$, square matrix $\mathbf{M}_{G}$ and column vector $\eta_{G}$ are defined as follows:

$$
\pi_{G}=\left(\lg \left(\rho_{1}(\omega)\right), \ldots, \lg \left(\rho_{k}(\omega)\right)\right)
$$

$\mathbf{M}_{G}=\left(m_{i j}\right)_{k \times k}$ where

$$
m_{i j}=\lg \left(\rho_{j}\left(\delta\left(a_{i}\right)\right)\right) \quad(\text { for } i, j=1, \ldots, k)
$$

and $\eta_{G}$ is a $k$-vector all entries of which are equal to 1 .

Theorem 1. Let $G$ be a DOL system. Then

(i) $f_{G}(n)=\pi_{G} \mathbf{M}_{G}^{n} \eta_{G} \quad$ (for $\left.n \in N\right)$;

(ii) the generating function $F_{G}$ of $f_{G}$ (i.e., the formal power series $F_{G}(x)=$ $\left.\sum_{n=0}^{\infty} f_{G}(n) x^{n}\right)$ is given by

$$
F_{G}(x)=\pi_{G}\left(\mathbf{I}-x \mathbf{I}_{G}\right)^{-1} \eta_{G}
$$

where $\mathbf{I}$ is an identity matrix of the same size as $\mathbf{M}_{G}$;

(iii) $f_{G}$ satisfies the linear homogeneous difference equation $P_{G}(D) f(n)=0$ where $P_{G}$ is the characteristic polynomial of $\mathbf{I}_{G}$ and $D$ is the transition operator given by

$$
D f(n)=f(n+1) \quad(\text { for } n \in N) .
$$

Proof. The proof of (i) is by easy induction. The claims (ii) and (iii) then follow from the formal identity

$$
\left(\mathbf{I}-x \mathbf{M}_{G}\right)^{-1}=\sum_{n=0}^{\infty} x^{n} \mathbf{M}_{G}^{n}
$$

(note that $\mathbf{I}-x \mathbf{M}_{G}$ is non-singular) and the Cayley-Hamilton Theorem. 
By part (ii) of the previous theorem the generating function of a DOL growth function is a rational function. In fact

$$
\begin{aligned}
\pi_{G}\left(\mathbf{I}-x \mathbf{M}_{G}\right)^{-1} \eta_{G} & =\frac{\pi_{G}\left|\mathbf{I}-x \mathbf{M}_{G}\right|\left(\mathbf{I}-x \mathbf{I}_{G}\right)^{-1} \eta_{G}}{\left|\mathbf{I}-x \mathbf{M}_{G}\right|} \\
& =\frac{\pi_{G}\left|\mathbf{I}-x \mathbf{M}_{G}\right|\left(\mathbf{I}-x \mathbf{M}_{G}\right)^{-1} \eta_{G}}{x^{k} P_{G}\left(\frac{1}{x}\right)}
\end{aligned}
$$

where $\left|\mathbf{I}-x \mathbf{M}_{G}\right|$ is the determinant of $\mathbf{I}-x \mathbf{M}_{G}$ and the alphabet of $G$ has $k$ elements.

Example 2. Consider the PDOL system $G=(\{a, b\}, \delta, a)$ where $\delta$ is given by $\delta(a)=b, \delta(b)=a b$. Then $\pi_{G}=(1,0)$,

and

$$
\mathbf{M}_{G}=\left(\begin{array}{ll}
0 & 1 \\
1 & 1
\end{array}\right), \eta_{G}=\left(\begin{array}{l}
1 \\
1
\end{array}\right)
$$

$$
F_{G}: F_{G}(x)=\frac{1}{1-x-x^{2}} \text {. }
$$

So $f_{G}(n)$ is the $n^{\text {th }}$ Fibonacci number (for $n \in N$ ).

We now state the DOL (resp. PDOL) growth synthesis problem for a set $S$ of functions from $N$ into $N$ : Find an algorithm (if there exists one) which, for each $f \in S$, determines whether or not there exist DOL (resp. PDOL ) systems $G$ such that $f=f_{G}$ and, should the answer be affirmative, gives $\pi_{G}$ and $\mathbf{M}_{G}$ for some such $G$.

The following two theorems give us some permissible operations on DOL (resp. PDOL) growth functions and their generating functions.

Theorem 2. A function (resp. non-decreasing function) $f: N \rightarrow N$ is a DOL (resp. PDOL) growth function if and only if, for an arbitrary $k \in N$ such that $f(0), \ldots, f(k-1) \neq 0$ if $f \neq 0$, also $\bar{f}: N \rightarrow N: \bar{f}(n)=$ $f(n+k)$ is a DOL (resp. PDOL) growth function.

Proof. $1^{\circ}$ Let $f: f(n)=\pi_{G} \mathbf{M}_{G}^{n} \eta_{G}$ for some DOL system $G$. Then $\bar{f}: \bar{f}(n)=\left(\pi_{G} \mathbf{M}_{G}^{k}\right) \mathbf{M}_{G}^{n} \eta_{G}$.

$2^{\circ}$ Let $\vec{f}: \vec{f}(n)=\pi_{G} \mathbf{M}_{G}^{n} \eta_{G}$ for some DOL system $G$. The theorem is trivial if $k=0$. So let $k \geq 1$. It is easily seen that, if $k=1$, then

$$
f: f(n)=\left(\begin{array}{l|l|l|l}
1|0| f(0)-1
\end{array}\right)\left(\begin{array}{c|c|c}
0 & \pi_{G} & 0 \\
\hline 0 & \mathbf{M}_{G} & 0 \\
\hline 0 & 0 & 0
\end{array}\right)^{n}\left(\begin{array}{c}
\frac{1}{\eta_{G}} \\
\hline 1
\end{array}\right)
$$

where the 0 's are zero matrices of appropriate sizes, and that, if $k>1$, then 


$$
f: f(n)=\left(\begin{array}{l}
1|0| f(0)-1
\end{array}\right)\left(\begin{array}{c|c|c|c}
0 & \mathbf{I} & 0 & \mathbf{F} \\
\hline 0 & 0 & \pi_{G} & 0 \\
\hline 0 & 0 & \mathbf{M}_{G} & 0 \\
\hline 0 & 0 & 0 & 0
\end{array}\right)^{n}\left(\begin{array}{c}
\eta \\
\hline \eta_{G} \\
\hline 1
\end{array}\right)
$$

where $\mathbf{I}$ is a $(k-1) \times(k-1)$-identity matrix, $\mathbf{F}=(f(1)-1, \ldots$, $f(k-1)-1)^{T}$ and $\eta$ is a $k$-vector all entries of which are equal to 1 .

The bracketed claim is proved analogously, only in part $2^{\circ}$ of the proof we may need matrices of sizes greater than above.

Theorem 3. Let $G$ and $\vec{G}$ be DOL (resp. PDOL) systems. Then the following results are valid:

(i) (originating from $A$. Szilard [7]) $f_{G}+f_{G}=f_{G^{\prime}}$ (and hence $F_{G}+F_{\bar{G}}=$ $\left.F_{Q^{\prime}}\right)$ for some DOL (resp. PDOL) system $G^{\prime}$;

(ii) (originating from $A$. Szilard [7]) for a $k \in N, k f_{G}=f_{G^{\prime}}$ (and hence $k F_{G}=F_{G^{\prime}}$ ) for some DOL (resp. PDOL, if $k>0$ ) system $G^{\prime}$;

(iii) (originating from A. Szilard [8]) $f_{G} \cdot f_{\bar{Q}}=f_{G^{\prime}}$ (and hence $F_{G} \odot F_{\bar{Q}}=$ $F_{G^{\prime}}$, where

$$
F_{G} \odot F_{\bar{G}}: \quad\left(F_{G} \odot F_{\bar{G}}\right)(x)=\sum_{n=0}^{\infty}\left(f_{\bar{Q}} \cdot f_{\bar{G}}\right)(n) x^{n}
$$

is the Hadamard product of $F_{G}$ and $F_{\bar{Q}}$ ) for some DOL (resp. PDOL) system $G^{\prime}$;

(iv) $F_{G} \cdot F_{\bar{G}}=F_{G^{\prime}}$ for some DOL (resp. PDOL) system $G^{\prime}$ (independently of the author this has been proved by $J$. Berstel [1]);

(v) (originating from $J$. Berstel [1]) $F_{G}^{*}=F_{G^{*}}$, where

$$
F_{G}^{*}: \quad F_{G}^{*}(x)=\frac{1}{1-x F_{G}(x)}
$$

is the quasi-inverse of $F_{G}$, for some DOL (PDOL system, if $\left.F_{G} \neq 0\right) G^{\prime}$.

Proof. (i) It is immediately seen that

$$
f_{G^{\prime}}: \quad f_{G^{\prime}}(n)=\left(\pi_{G} \mid \pi_{\bar{G}}\right)\left(\begin{array}{c|c}
\mathbf{M}_{G} & \mathbf{0} \\
\hline 0 & \mathbf{M}_{\bar{G}}
\end{array}\right)^{n}\left(\begin{array}{c}
\eta_{\bar{G}} \\
\hline \eta_{\bar{G}}
\end{array}\right) .
$$

(ii) It is immediately seen that

$$
f_{G^{\prime}}: \quad f_{G^{\prime}}(n)=\left(k \pi_{G}\right) \mathbf{M}_{G}^{n} \eta_{G} .
$$

(iii) For any matrices $\mathbf{A}=\left(a_{i j}\right)_{p \times q}$ and $\mathbf{B}$ we denote by $\mathbf{A} \odot \mathbf{B}$ the Hadamard product of $\mathbf{A}$ and $\mathbf{B}$ i.e., 


$$
\mathbf{A} \odot \mathbf{B}=\left(\begin{array}{c|c|c}
a_{11} \mathbf{B} & \cdots & a_{1 q} \mathbf{B} \\
\hline \vdots & & \vdots \\
\hline a_{p 1} \mathbf{B} & \ldots & a_{p q} \mathbf{B}
\end{array}\right) .
$$

Then

$$
\begin{aligned}
f_{G^{\prime}}: \quad f_{G^{\prime}}(n) & =\left(\pi_{G} \odot \pi_{\bar{G}}\right)\left(\mathbf{M}_{G} \odot \mathbf{M}_{\bar{G}}\right)^{n}\left(\eta_{G} \odot \eta_{\bar{G}}\right) \\
& =\left(\pi_{G} \odot \pi_{\bar{G}}\right)\left(\mathbf{M}_{G}^{n} \odot \mathbf{M}_{\bar{G}}^{n}\right)\left(\eta_{G} \odot \eta_{\bar{G}}\right) \\
& =\left(\pi_{G} \mathbf{M}_{G}^{n} \eta_{G}\right) \odot\left(\pi_{\bar{G}} \mathbf{M}_{\bar{G}}^{n} \eta_{\bar{G}}\right)=f_{G}(n) f_{\bar{G}}(n) .
\end{aligned}
$$

(iv) By easy induction we see that

$$
\begin{aligned}
f: \quad f(n) & =\left(\pi_{G} \mid 0\right)\left(\frac{\mathbf{M}_{G}}{0} \mid \frac{\eta_{G} \odot\left(\pi_{\bar{G}} \mathbf{M}_{\bar{G}}\right)}{\mathbf{M}_{\bar{G}}}\right)^{n}\left(\frac{\eta_{G}}{\eta_{\bar{G}}}\right) \\
& =\left(\pi_{G} \mid 0\right)\left(\frac{\mathbf{M}_{G}^{n}}{0} \mid \frac{\sum_{i=0}^{n-1} \mathbf{M}_{\bar{G}}^{n-1-i}\left(\eta_{G} \odot\left(\pi_{\bar{G}} \mathbf{M}_{\bar{G}}\right)\right) \mathbf{M}_{\bar{G}}^{i}}{\mathbf{M}_{\bar{G}}^{n}}\right)\left(\frac{\eta_{G}}{\eta_{\bar{G}}}\right) \\
& =\pi_{G} \mathbf{M}_{G}^{n} \eta_{G}+\sum_{i=0}^{n-1} \pi_{G} \mathbf{M}_{G}^{n-1-i}\left(\eta_{G} \odot\left(\pi_{\bar{G}} \mathbf{M}_{\bar{G}}\right)\right) \mathbf{M}_{\bar{G}}^{i} \eta_{\vec{G}} \\
& =f_{G}(n)+\sum_{i=0}^{n-1}\left(\pi_{G} \mathbf{M}_{\bar{G}}^{n-1-i} \eta_{G}\right) \odot\left(\pi_{\bar{G}} \mathbf{M}_{\bar{G}}^{i+1} \eta_{\bar{G}}\right) \\
& =f_{G}(n)+\sum_{i=0}^{n-1} f_{G}(n-1-i) f_{\bar{G}}(i+1) \\
& =f_{G^{\prime}}(n)-\left[f_{\bar{G}}(0)-1\right] f_{G}(n)
\end{aligned}
$$

and so $f_{Q^{\prime}}=f+\left[f_{\bar{G}}(0)-1\right] f_{G}$. By (i) and (ii) the claim now follows.

(v) Since

$$
\begin{aligned}
F_{G}^{*}(x) & =\frac{1}{1-x \pi_{G}\left(\mathbf{I}-x \mathbf{M}_{G}\right)^{-1} \eta_{G}} \\
& =1+x \frac{\pi_{G}\left(\mathbf{I}-x \mathbf{M}_{G}\right)^{-1} \eta_{G}}{1-x \pi_{G}\left(\mathbf{I}-x \mathbf{M}_{G}\right)^{-1} \eta_{G}}
\end{aligned}
$$

it suffices to show (cf. Theorem 2) that

$$
H: \quad H(x)=\frac{\pi_{G}\left(\mathbf{I}-x \mathbf{M}_{G}\right)^{-1} \eta_{G}}{1-x \pi_{G}\left(\mathbf{I}-x \mathbf{M}_{G}\right)^{-1} \eta_{G}}
$$

is $F_{G^{\prime \prime}}$ for some DOL system $G^{\prime \prime}$. Denote $\overline{\mathbf{M}}=\mathbf{M}_{G}+\eta_{G} \pi_{G}$. So

$$
\mathbf{I}-x \overline{\mathbf{M}}=\mathbf{I}-x \mathbf{M}_{G}-x \eta_{G} \pi_{G} .
$$

Multiplying by $\pi_{G}\left(\mathbf{I}-x \mathbf{M}_{G}\right)^{-1}$ and $(\mathbf{I}-x \overline{\mathbf{M}})^{-1} \eta_{G}$ we get

$$
\begin{gathered}
\pi_{G}\left(\mathbf{I}-x \mathbf{M}_{G}\right)^{-1} \eta_{G}= \\
\pi_{G}(\mathbf{I}-x \overline{\mathbf{M}})^{-1} \eta_{G}-x \pi_{G}\left(\mathbf{I}-x \mathbf{M}_{G}\right)^{-1} \eta_{G} \pi_{G}(\mathbf{I}-x \overline{\mathbf{M}})^{-1} \eta_{G} .
\end{gathered}
$$


Hence $H(x)=\pi_{G}(\mathbf{I}-x \overline{\mathbf{M}})^{-\mathbf{1}} \eta_{G}$. The claim now follows when we take $\pi_{G^{\prime \prime}}=\pi_{G}$ and $\mathbf{M}_{G^{\prime \prime}}=\overline{\mathbf{M}}$ (cf. Theorem 1 (ii)).

It is easily seen that the bracketed claim also holds true.

\section{A class of DOL growth functions}

We define a class of DOL growth functions recursively in terms of their generating functions.

Theorem 4. Let $\alpha_{1}, \ldots, \alpha_{k}$ be integers such that

$$
\sum_{i=1}^{j} \alpha_{i}>0 \quad(\text { for } j=1, \ldots, k) .
$$

Then

$$
F: \quad F(x)=\frac{1}{1-\alpha_{1} x-\ldots-\alpha_{k} x^{k}}
$$

is $F_{G}$ for some $P D O L$ system $G$. I.e., there is a PDOL system $G$ such that $f_{G}$ satisfies the difference equation

$$
f(n+k)=\sum_{i=1}^{k} \alpha_{i} f(n+k-i)
$$

with initial values $f(-k+1)=\ldots=f(-1)=0, f(0)=1$.

Proof. The equivalence of the last two sentences of the theorem is an easy verification.

If $k=1$, then obviously

$$
f_{G}: \quad f_{G}(n)=(1)\left(\alpha_{1}\right)^{n}(1) .
$$

Suppose that $k \geq 2$. Let $f$ be the function which satisfies the difference equation (2) with the given initial values. Consider the $k$-vector $\pi=$ $(1,0, \ldots, 0)$ and the $k \times k$-matrix

$$
\mathbf{M}=\left(\begin{array}{c|c}
\mathbf{A} & \mathbf{I} \\
\hline \sum_{i=1}^{k} \alpha_{i}-\mathbf{1} & \mathbf{B}
\end{array}\right)
$$

where $\mathbf{A}=\left(\alpha_{1}-1, \ldots, \sum_{i=1}^{k-1} \alpha_{i}-1\right)^{T}$ and $\mathbf{B}=(0, \ldots, 0,1)$. We claim that $\pi \mathbf{M}^{n}=\pi_{n}$, where

$$
\pi_{n}=(f(n)-f(n-1), \ldots, f(n-k+2)-f(n-k+1), f(n-k+1))
$$

(for $n \in N$ ). We prove the claim by induction. Obviously $\pi_{0}=\pi$. Suppose that $\pi \mathbf{M}^{n}=\pi_{n}$ for some $n \in N$. Then 


$$
\begin{aligned}
\pi \mathbf{M}^{n+1}= & \pi_{n} \mathbf{M}=\left([f(n)-f(n-1)]\left(\alpha_{1}-1\right)+\ldots\right. \\
& +[f(n-k+2)-f(n-k+1)]\left(\sum_{i=1}^{k-1} \alpha_{i}-1\right)+ \\
& f(n-k+1)\left(\sum_{i=1}^{k} \alpha_{i}-1\right), \\
& f(n)-f(n-1), \ldots, f(n-k+3)-f(n-k+2), f(n-k+2)) \\
= & \left(\sum_{i=1}^{k} \alpha_{i} f(n-i+1)-f(n), f(n)-f(n-1), \ldots,\right. \\
& f(n-k+3)-f(n-k+2), f(n-k+2))=\pi_{n+1} .
\end{aligned}
$$

The theorem now immediately follows when we choose $\pi_{G}=\pi$ and $\mathbf{M}_{G}=\mathbf{M}$.

Example 3. By Theorem 4

$$
F: \quad F(x)=\frac{1}{1-10 x+2 x^{2}-24 x^{3}+2 x^{5}}
$$

is the generating function of some PDOL growth function. We may note in passing that the characteristic equation of the corresponding difference equation cannot be solved by radicals.

Theorem 5. Let $\alpha_{1}, \ldots, \alpha_{k}$ be as in Theorem 4 and $l, c_{0}, \ldots, c_{l-1}$ be positive integers. Then

$$
F: \quad F(x)=\frac{c_{0}+c_{1} x+\ldots+c_{l-1} x^{l-1}}{1-\alpha_{1} x^{l}-\alpha_{2} x^{2 l}-\ldots-\alpha_{k} x^{k l}}
$$

is $F_{G}$ for some DOL system $G$. I.e., there is a DOL system $G$ such that $f_{G}$ satisfies the difference equation

$$
f(n+k l)=\sum_{i=1}^{k} \alpha_{i} f(n+(k-i) l)
$$

with initial values $f(-(k-1) l)=\ldots=f(-1)=0$ and $f(i)=c_{i} \quad$ (for $i=0, \ldots, l-1$ ). Moreover, if $l>1$, there is a column vector $\mu_{G}$ (of appropriate size) all entries of which are in $\{0,1\}$ such that

$$
H: \quad H(x)=\frac{x^{l-1}}{1-\alpha_{1} x^{l}-\alpha_{2} x^{2 l}-\ldots-\alpha_{k} x^{k l}}
$$

is the generating function of $h: h(n)=\pi_{G} \mathbf{M}_{G}^{n} \mu_{G}$.

Proof. The equivalence of the second and the third sentence of the theorem is an easy verification.

If $l=1$, the theorem follows immediately from Theorem 4 and part (ii) of Theorem 3. Suppose then that $l \geq 2$. Let $\bar{G}$ be the PDOL system for which

$$
F_{\bar{G}}: \quad F_{\bar{G}}(x)=\frac{1}{1-\alpha_{1} x-\ldots-\alpha_{k} x^{k}}
$$

given by Theorem 4. Consider the $(k l+1)$-vector 


$$
\pi=\left(\pi_{\bar{G}}|0| \ldots|0|\left(c_{0}-1\right) \pi_{\bar{G}} \eta_{\bar{G}}\right)
$$

where the 0 's are zero matrices of size $1 \times k$, and the $(k l+1) \times(k l+1)$ matrix

$$
\mathbf{M}=\left(\begin{array}{c|c|c}
\mathbf{0} & \mathbf{I} & \mathbf{A} \odot \eta_{\bar{G}} \\
\hline \mathbf{M}_{\bar{G}} & \mathbf{0} & \left(c_{0}-1\right) \mathbf{M}_{\bar{G}} \eta_{\bar{G}} \\
\hline \mathbf{0} & \mathbf{0} & 0
\end{array}\right)
$$

where $\mathbf{A}=\left(c_{1}-1, \ldots, c_{l-1}-1\right)^{T}$. By straightforward induction we can show that

$$
\pi \mathbf{M}^{l q+r}=\underbrace{(0|\ldots| 0 \mid}_{r \text { copies }} \pi_{\bar{G}} \mathbf{M}_{l-r-1 \text { copies }}^{q} \underbrace{|0|}_{l-r|\ldots| 0 \mid}\left(c_{r}-1\right) f_{\bar{G}}(q))
$$

(for $q \in N$ and $r \in\{0, \ldots, l-1\}$ ) where the 0 's are zero matrices of size $1 \times k$. Hence the theorem follows when we choose $\pi_{G}=\pi, \mathbf{M}_{G}=\mathbf{M}$ and

$$
\mu_{G}=\underbrace{(0|\ldots| 0 \mid}_{l-1 \text { copies }} \eta_{G}^{T} \mid 0)^{T}
$$

where the 0 's are zero matrices of size $1 \times k$.

The DOL system $G$ in the previous theorem is not propagating (unless $l=1$ ). In case $c_{i}>c_{i+1}$ for some $i \in\{0, \ldots, l-2\}$ or $c_{l-1} f_{\bar{G}}(n)>c_{0} f_{\bar{G}}(n+1)$ for some $n \in N$, there clearly is no such PDOL system $G^{\prime}$ that $f_{G}=f_{G^{\prime}}$. Otherwise the question whether or not $f_{G}=f_{G^{\prime \prime}}$ for some PDOL system $G^{\prime \prime}$, remains open (except when $l=1$ ).

Theorem 6. Let $\alpha_{1}, \ldots, \alpha_{k}$ be as in Theorem 4, $l$ be a positive integer and $G$ a DOL system such that $f_{G}(0), \ldots, f_{G}(l-1)>0$. Then

$$
F: \quad F(x)=\frac{F_{G}(x)}{1-\alpha_{1} x^{l}-\alpha_{2} x^{2 l}-\ldots-\alpha_{k} x^{k l}}
$$

is $F_{\bar{G}}$ for some DOL system $\bar{G}$.

Proof. If $l=1$, the theorem follows from Theorem 4 and part (iv) of Theorem 3. So suppose $l \geq 2$. Let $G^{\prime}$ be the DOL system, given in Theorem 5, for which

$$
F_{G^{\prime}}: \quad F_{G^{\prime}}(x)=\frac{f_{G}(0)+f_{G}(1) x+\ldots+f_{G}(l-1) x^{l-1}}{1-\alpha_{1} x^{l}-\alpha_{2} x^{2 l}-\ldots-\alpha_{k} x^{k l}} .
$$

Then

$$
Q: \quad Q(x)=\frac{x^{l-1}}{1-\alpha_{1} x^{l}-\alpha_{2} x^{2 l}-\ldots-\alpha_{k} x^{k l}}
$$


is the generating function of $q: q(n)=\pi_{G^{\prime}} \mathbf{M}_{G^{\prime}}^{n} \mu_{G^{\prime}}$. Consider the row vector $\pi=\left(\pi_{G^{\prime}} \mid 0\right)$, where the 0 is of the same size as $\pi_{G}$, and the square matrix

$$
\mathbf{M}=\left(\frac{\mathbf{M}_{G^{\prime}}}{\mathbf{0}} \mid \frac{\mu_{G^{\prime}} \odot \pi_{G} \mathbf{M}_{G}^{l}}{\mathbf{M}_{G}}\right) \text {. }
$$

Then (cf. the proof of part (iv) of Theorem 3)

$$
\pi \mathbf{I}^{n}\left(\frac{\eta_{G^{\prime}}}{\eta_{G^{\prime}}}\right)=f_{G^{\prime}}(n)+\sum_{i=1}^{n} q(n-i) f_{G}(l+i-1)
$$

(for $n \in N$ ) and so

$$
\begin{aligned}
\sum_{n=0}^{\infty} \pi \mathbf{I}^{n}\left(\frac{\eta_{G^{\prime}}}{\eta_{G}}\right) x^{n} & =F_{G^{\prime}}(x)+Q(x) \sum_{n=1}^{\infty} f_{G}(l+n-1) x^{n} \\
& =F(x) .
\end{aligned}
$$

Hence the theorem follows when we choose $\pi_{\bar{G}}=\pi$ and $\mathbf{M}_{\bar{G}}=\mathbf{M}$.

Starting from the zero function and then using repeatedly the operations given in Theorems 2 (considered as operations on generating functions), 3 and 6 , we get a wide class $C$ of generating functions of synthetizable DOL growth functions.

Correspondingly, starting from the generating functions given in Theorem 4 and using repeatedly the operations of Theorems 2 (the bracketed version) and 3 we get a class $C_{\text {prop }}$ of generating functions of synthetizable PDOL growth functions.

Some questions now naturally arise:

(i) Is $\mathrm{C}$ wide enough to contain the generating functions of all DOL growth functions?

(ii) Does $C_{\text {prop }}$ contain the generating functions of all PDOL growth functions?

(iii) Should the answers to questions (i) and (ii) be negative, are there generating functions of PDOL growth functions in $\mathrm{C} \sim \mathrm{C}_{\text {prop }}$ ? (Cf. the discussion after Theorem 5.)

(iv) Is it sufficient to take

$$
F: \quad F(x)=\frac{1}{1-x}
$$

as a starting function for $C_{\text {prop }}$ ?

We do not have the answer to any of these questions. 


\section{A subclass: polynomially bounded DOL growth functions}

We show that the class $\mathrm{C}$ is wide enough to contain the generating functions of all polynomially bounded DOL growth functions, i.e. DOL growth functions $f$ such that

$$
f(n) \leq P(n) \quad(\text { for } n \in N)
$$

for some polynomial $P$.

We denote by $S_{1}$ the set of functions from $N$ into $N$ given by the following conditions (i)-(iii).

(i) The zero function is in $S_{1}$.

(ii) $S_{1}$ contains all functions $g$ from $N$ into the set of positive integers of the form

$$
g(n)=\sum_{t=1}^{T} P_{t}(n) r_{t}(n) \quad(\text { for } n \in N)
$$

where $P_{1}, \ldots, P_{T}$ are polynomials, $r_{1}, \ldots, r_{T}$ are periodical functions, with periods $j_{1}, \ldots, j_{T}$ respectively, and the polynomials

$$
R_{i}: \quad R_{i}(n)=g(n J+i) \quad(\text { for } i=0, \ldots, J-1),
$$

where $J=\operatorname{lcm}\left(j_{1}, \ldots, j_{T}\right)$, are all of the same degree (we denote the degree of a polynomial $P$ by $G[P]$; by convention $G[0]=-1$ ).

(iii) In addition to the functions given in (i) and (ii), $S_{1}$ contains all functions which we get from them by applying the operations given in Theorem 2.

Theorem 7. All polynomially bounded DOL growth functions are in $S_{1}$.

Proof. Let $G$ be a DOL system such that $f_{G}$ is polynomially bounded. J. Karhumäki [4] has shown-using a result of J. Berstel's-that then we can choose $G$ in such a way that the roots $\rho_{1}, \ldots, \rho_{k}$ of the characteristic equation of the associated difference equation $P_{G}(D) f(n)=0$ are all in the unit circle. By the well-known Kronecker Theorem each $\rho_{i}$ is then either zero or equal to some root of unity. Thus $f_{G}$ is of the desired form (the zero roots correspond to the use of the operations of Theorem 2). The only thing still to be shown is that the associated polynomials $R_{0}$, $\ldots, R_{J-1}$ are of the same degree. Indeed, if $G\left[R_{i}\right]>G\left[R_{j}\right]$ for some $i, j=0, \ldots, J-1$ such that $i \neq j$, then

$$
\lim _{n \rightarrow \infty} \frac{R_{i}(n)}{R_{j}(n)}=\lim _{n \rightarrow \infty} \frac{f_{G}(n J+i)}{f_{G}(n J+j)}=\infty
$$


(clearly the leading coefficients of $R_{i}$ and $R_{j}$ must be positive) which is impossible since the ratio $f_{G}(n+i) / f_{G}(n+j)$ is bounded.

The next theorem shows that the generating functions of the functions of $S_{1}$ are in $C$. Since these functions obviously are polynomially bounded, it then follows that $S_{1}$ is equal to the class of all polynomially bounded DOL growth functions.

Theorem 8. The generating functions of the functions of $S_{1}$ are in $C$.

Proof. By Theorem 2 it suffices to prove the claim for those functions of $S_{1}$ which are defined by (i) and (ii). The proof is by induction on the common degree $m$ of the associated polynomials $R_{0}, \ldots, R_{J-1}$. The case $m=-1$ is trivial. So suppose $m>-1$. Then the polynomials are either constant polynomials or increasing when restricted onto some set $\{n \mid n \in N, n \geq l\}$. By Theorem 2 it suffices to show that $\bar{g}: \bar{g}(n)=$ $g(n+l)$ is $f_{G}$ for some DOL system $G$. We see that

$$
\begin{gathered}
\sum_{n=0}^{\infty} \bar{g}(n) x^{n}=\frac{\left(1-x^{J}\right) \sum_{n=0}^{\infty} \bar{g}(n) x^{n}}{1-x^{J}} \\
=\frac{\bar{g}(0)+\bar{g}(1) x+\ldots+\bar{g}(J-1) x^{J-1}+x^{J} \sum_{n=0}^{\infty}[\bar{g}(n+J)-\bar{g}(n)] x^{n}}{1-x^{J}}
\end{gathered}
$$

and, if we denote

$$
R_{i}(n)=a_{i, m} n^{m}+a_{i, m-1} n^{m-1}+\ldots+a_{i, 0} \quad(\text { for } \quad i=0, \ldots, J-1),
$$

that

$$
R_{i}(n+1)-R_{i}(n)=m a_{i, m} n^{m-1}+\ldots+\left(a_{i, m}+\ldots+a_{i, 1}\right)
$$

(for $i=0, \ldots, J-1)$. Thus the function $h: h(n)=\bar{g}(n+J)-\bar{g}(n)$ is in $S_{1}$ and the common degree of the associated polynomials is $m-1$. The claim now follows from Theorem 6 .

Should a function $g \in S_{1}$ be ultimately polynomial (i.e., we get $g$ from some polynomial by using the operations of Theorem 2), the above proof (instead of Theorem 6 we now use Theorem 4) shows that $g$ is ultimately a PDOL growth function (i.e., we get $g$ also from some PDOL growth function by using the operations of Theorem 2 (the non-bracketed version)). This is a well-known result (see Szilard [7]) and we have given a new proof for it.

\section{Acknowledgement}

The author is indepted to Mr. J. Karhumäki for many helpful discussions during the preparation of this paper. 


\section{References}

[1] Bersted, J,: Personal communication.

[2] Doucet, P.: The growth of word length in DOL systems. - University of Aarhus, Computer Science Department, Technical Report No. 15, 1973, 83-94.

[3] Lindenmayer, A.: Mathematical models for cellular interactions in development I, II. - J. Theoret. Biol. 18, 1968, 280-315.

[4] Karhumäki, J.: On length sets of L systems. - Licentiate Thesis, University of Turku, 1974.

[5] PAz, A., and A. SAlomaA: Integral sequential word functions and growth equivalence of Lindenmayer systems. - Information and Control 23, 1973, $313-343$

[6] SAlomaA, A.: On exponential growth in Lindenmayer systems. - Indag. Math. 35, $1973,23-30$.

[7] SzIlard, A.: Growth functions of Lindenmayer systems. - University of Western Ontario, Computer Science Department, Technical Report No. 4, 1971.

[8] SzILARD, A.: Unpublished results.

[9] Vitánxi, P.: Structure of growth in Lindenmayer systems. - Indag. Math. 35, 1973, 247-253.

University of Turku

Department of Mathematics

SF-20500 Turku 50

Finland

Received 28 November 1974 